\title{
Valuing the impact of Self-rated Health and Social Support on life satisfaction among Hong Kong Chinese population
}

Chee Hon Chan ( $\sim$ gchc@hku.hk)

University of Hong Kong

\section{Research Article}

Keywords: Wellbeing valuation, self-rated health, social support, willingness-to-pay, social impact assessment, Chinese

Posted Date: February 12th, 2021

DOI: https://doi.org/10.21203/rs.3.rs-155505/v1

License: (a) (i) This work is licensed under a Creative Commons Attribution 4.0 International License.

Read Full License 
1 Valuing the impact of Self-rated Health and Social Support on life satisfaction among Hong Kong

\section{Chinese population}

3

4 Author:

5 Chee Hon, CHAN, $\mathrm{PhD}^{1,2}$

6

7 Corresponding author

$8 \quad$ Email: gchc@hku.hk

9 ORCID iD: $\underline{0000-0002-4112-9383}$

10

$11{ }^{1}$ Faculty of Social Sciences, The University of Hong Kong, Pokfulam, Hong Kong

$12{ }^{2}$ The HKJC Centre for Suicide Research and Prevention, The University of Hong Kong, Pokfulam, 13 Hong Kong

14

15

16

17

18

19

20

21

22

23

24

25

26

27 


\section{Abstract}

Background: Research has highlighted that satisfaction in health and social support are key areas of life affecting individual's wellbeing. Many social and public health initiatives use these two intervention mechanisms to improve individual's wellbeing. For the purpose of cost-benefit assessment, there has been growing interest in expressing these intervention effects in economic terms. However, only a handful of studies have ever estimated these effects in economic terms, and none of which examined in a Chinese context. The aim of this study is to extend this line of valuation work to estimate the implicit willingness-to-pays on the effects of improving individuals' self-rated health status (SRH) and social support (SS) on their life satisfaction in the Chinese population.

Methods: Using individual's life satisfaction data from a two-wave representative panel survey in Hong Kong $(\mathrm{n}=1,109)$, this study first conducted a cross-lagged analysis with structural equation modelling technique to examine the causal effects of SRH and SS on life satisfaction, while simultaneously adjusting their reverse causal influences. The use of this cross-lagged approach was the effort to minimising the endogeneity problem. Then, substituting the respective estimates to the formulae of compensating surplus, the marginal rate of substitution of SRH and SS with respect to individual's equivalised monthly household were estimated and were then expressed as the willingness-to-pays on the effect of improving individuals' SRH and SS on their life satisfaction.

Results: The cross-lagged analysis ascertained the casual effects of SRH ( $\beta=0.078,95 \%$ CI: 0.020 , 0.151 ) and SS on individuals' satisfaction with life. The sample's marginal rate of substitution of $S R H$ and $S S$ were found to be 1.28 (95\%CI: $0.43,2.15)$ and 1.36 (95\%CI: $0.23,2.49)$ respectively. Translating into the concept of compensating surplus, the implicit monetary values of improving the sample's $S R H$ from "poor health" to "excellent health" and their $S S$ from "little support" to "a lot of support" are equivalent to an increase in their equivalised monthly household income by HK $\$ 56,000$ and $\mathrm{HK} \$ 39,400$ respectively. 
Conclusions: This study has implications for the cost-benefit assessment in wellbeing initiatives for the Chinese population.

57

58 Keywords: Wellbeing valuation, self-rated health, social support, willingness-to-pay, social impact assessment, Chinese

60

Valuing the impact of Self-rated Health and Social Support on life satisfaction among Hong Kong

\section{Chinese population}

63

\section{Background}

65

66

67

In recent years the concept of individuals' wellbeing has increasingly taken centre stage in public policy discussion. An increasing number of policy thinkers have emphasised a key objective of health and social policy is to improve individuals' wellbeing and collectively raise population welfare [1-4]. Worldwide, policy initiatives to enhance population wellbeing have been growing, such as enhancing access to health care and building social support and community cohesion. Interest in assessing the effectiveness of the wellbeing policy has been stacking up, and the preference to capture the impact in some form of economic cost-benefit analysis is common $[5,6]$.

While cost-benefit analysis has been used in a wide array of policy domains, it can be challenging when applied to wellbeing policy, since impacts of many of these initiatives such as improving health status and social support are difficult to measure in economic terms (i.e., non-market goods without direct monetary value). Previous research has developed valuation methods to resolve this challenge, either through the revealed-preference approach or the stated-preference approach [710]. However, through decades of research, it is known that multiple factors in the valuation process, such as the cognitive biases caused either by the experimental settings or due to a non-equilibrium market, could affect the valuation decision and hence leads to inadequate estimates [11-15].

Against this backdrop, recent research has developed the use of subjective wellbeing data, such as life satisfaction, as an alternative non-market valuation approach $[13,16,17]$. In this context, 
measures of life satisfaction that capture individuals' appraisals of their overall quality of life with regards to past experience, expectations of the future, and comparison to others [18], are considered to be indicators of one's utility [19]. Surveys that explore how exposure to some non-market circumstances could causally affect individuals' life satisfaction are then interpreted as a direct empirical approximation of how the goods of interest alter individual welfare. Then, by weighting against the causal effect of household income on individuals' life satisfaction, they together form the implicit willingness-to-pay (WTP), or the 'shadow price', of the goods of interest (i.e., additional income to pay or accept in order to compensate for the changes in satisfaction with life for the losses or gains in some particular conditions). Scholars have highlighted that this wellbeing valuation approach does not rest upon individuals' decisions in the valuation process, hence avoiding potential cognitive biases that could have been involved in the stated-preference and revealed-preference methods [20,21]. The use of this wellbeing valuation approach has gained considerable traction in recent years and has started to be applied in estimating the economic term for various health and social conditions. Internationally, a handful of studies have used this wellbeing valuation approach to estimate the effects of improving the self-rated status and social support on life satisfaction [20, 22-26]. However, the estimates derived from these studies varied considerably. For instance, a study in the United Kingdom estimated that the economic value of improving an individual's self-rated health from 'poor health' to 'excellent health' is equivalent to increasing his/her annual household income per capita $£ 303,000$ [22], other studies reported a substantially smaller estimate (e.g., US\$1,644 - \$1,692 from Chandoevwit \& Thampanishvong's study [25]; see Additional file 1). Some variation of the estimates may be methodologically related as some of the prior work ignored the endogeneity issue in their estimation $[22,26,27]$, which is a methodological concern that could introduce biases and cause an overestimation of the monetary value of the non-market goods $[27,28]$. Also, many of these existing valuation work were conducted in the Western setting. To the best of the author's knowledge, only two studies have used this wellbeing valuation approach to estimate the effects of improving health status and social relationship on life satisfaction in an Asian context [25, 26], and none of which examined in a Chinese context. Hence, to fill this research gap, the aim of this study is to extend the literature by using the 
wellbeing valuation method to estimate the implicit WTPs of the effects of improving individuals' selfrated health status and social support on their life satisfaction in the Chinese population.

The measure of individuals' satisfaction with life has been conceptualised as one of the major indicators of one's overall wellbeing $[29,30]$, and a substantial body of research from the domains of life literature has postulated that individuals' wellbeing depends on their appraisals on various aspects of daily life activities $[31,32]$. Previous research has highlighted that several areas such as health status and social relationships, and among some others (e.g., material wellbeing, work, community etc.) are the key areas that determine individuals' assessment of life [33, 34]. Self-rated health status, a measure of one's overall physical and mental health condition, has been shown to be related to individuals' satisfaction with life. Impaired health is associated with a decline in positive affect and an increase in negative feelings, such as a depressed mood and anger [35]. In addition, with worsening health, individuals' involvement in social activities is likely to be restricted, and they, therefore, have a limited social connection $[36,37]$. Extent research has also demonstrated that individuals having greater social support tended to have a higher level of life satisfaction. Social support, which refers to individuals' actual or perceived available social resources coming from their networks, such as family, friends, coworkers, and from other community ties, can enhance bonding and serve as a buffer for negative stressors. Social support as a protective factor has also been found to alleviate the burden of depressive symptoms, generate happiness, and boost overall life satisfaction $[38,39]$. This study focused on selfrated health and social support as they are intervention mechanisms commonly used to enhance individual welfare; deriving the monetary value of these two variables provides wide application in cost-benefit policy analysis.

\section{Methods}

\section{Sample}

Data used in this study were extracted from the Hong Kong Panel Survey for Poverty Alleviation, which is a two-wave household survey with a representative sample recruited through a stratified random sampling strategy by 412 geographical constituency area. The panel survey involved conducting face- 
to-face interviews with the household head to elicit a variety of information; Each interview lasted

137 approximately 60 minutes. Content of the survey and details of the sampling strategy has been reported 138 elsewhere [40]. The first wave of the panel survey was conducted between September 2015 and April 139 2016, in which 2,002 households were recruited. These households were interviewed again 12 months 140 after the first interview (the second wave), and 1,109 households were retained (retention rate: 50.2\%).

141 Given that a notable proportion of the sample recruited in the first wave was lost in the follow-up, the 142 Inverse Mills analysis was conducted to explore the potential sample selection bias, and the result did 143 not indicate a substantial concern (Inverse Mills Ratio: $1.66 ; \mathrm{p}=0.678$ ).

144 This study retrieved data on the sample's socioeconomic and demographic characteristics (i.e., 145 age, sex, educational attainment, marital status, employment status, housing tenure, household size, and monthly household income), and their status of self-rated health, social support, and satisfaction with

147 life. The sample's self-rated health status was assessed by a single item eliciting their overall health (1 $148=$ poor health to $5=$ excellent health). Previous studies have shown that this is a valid and reliable measure for one's overall physical and mental health condition [41], reflects participation in health risk

150 behaviours [42], and predicts mortality risk [41]. The sample's level of social support was assessed using three items exploring the levels of perceived available support from their family members and friends $(1=$ little support to $4=$ a lot of support $)$. Three types of social support were elicited, including instrumental support, informational support, and emotional support [43]. For life satisfaction, the fiveitem Chinese version of the Satisfaction with Life Scale (SWLS) was employed to elicit sample's satisfaction with life. The total scores of the SWLS range from 5 (extremely dissatisfied) to 35 (extremely satisfied). The Chinese version of the SWLS has been validated and was found to have

157 adequate validity and reliability [44].

\section{Statistical analyses}

160 Analysis in wellbeing valuation work can be generally conceptualised as having two-part. The first part involves a regression analysis estimating the effects of some non-market conditions (i.e., self-rated

162 health and social support) on life satisfaction and respectively the effect of an economic indicator (e.g., 163 household income) on life satisfaction. Estimates of these effects would then form the marginal rate of 
substitution of the non-market conditions with respect to the economic indicator. As mentioned earlier,

165 one major methodological concern that could introduce biases in this part of estimation is the 166 endogeneity issue [45] and failing to account it can cause an overestimation of the monetary value of 167 the non-market goods [27, 28]. Studies typically used instrumental variables to handle the problem, but the use of the instruments also has its own constraints. Specifically, variables that qualify as an instrument should fulfil the independence assumption, i.e., the instruments should not share causes with the outcome variables (e.g., life satisfaction). Researchers have highlighted that it is very difficult to identify appropriate instruments that meet this assumption [27], as "almost every factor determines life satisfaction" [46].

An alternative way to tackle the endogeneity issue in wellbeing valuation is the use of a crosslagged analysis [47]. A typical cross-lagged model would examine six effects between two variables. For instance, applying in an examination of the relationships between SRH and life satisfaction (LS), two reflects the temporal autoregressive effects of the variable of SRH and LS ( $\beta_{a}$ : the causal effects of $S R H_{1}$ on $S R H_{2}$ and $L S_{1}$ on $L S_{2}$, respectively) and two cross-sectional correlations between SRH and

178 LS ( $\beta_{\text {cor }}$ : the correlations between $S R H_{1}$ and $L S_{1}$ and $S R H_{2}$ and $L S_{2}$, respectively). The remaining two 179 effects examine the reciprocal nature between $S R H$ and $L S$, which is the causal effect of $S R H_{1}$ on $L S_{2}$ $180(\beta \mathrm{c})$ and the reverse causal effect of $L S_{1}$ on $S R H_{2}(\beta \mathrm{rc})$. of $S R H_{1}$ on $L S_{2}$ ), as it will be used for calculating the marginal rate of substitution. Specification of the other five effects are ways to reduce the endogeneity problem in the estimation process. Specifically, given the endogeneity bias typically arises from reverse causality $(\beta \mathrm{rc})$ and omitted influences on the dependent variable (e.g., temporal autoregressions, $\beta_{a}$, and cross-sectional correlations, $\beta_{c o r}$ ) [45], the cross-lagged model explicitly addressing them help to reduce these potential influences in the estimation of the causal effect of $S R H$ on $L S$. The cross-lagged model is computed based on a structural equation modelling (SEM) technique, which is a form of simultaneous regression technique often used in the work of subjective wellbeing valuation. Previous studies have used SEM to examine the causal 
determinates of life satisfaction [48], and recent valuation studies have used this technique in valuing environmental goods [46].

In this study, a cross-lagged model was specified to examine the relationships among the variables of SRH, SS, HI, and LS. The full model analysed all the effects between the pairs of HI, SRH, SS, and LS within and across the two timepoints. In addition, sample's sociodemographic characteristics were hypothesised as covariates and were regressed on the outcome variables (HI, SRH, SS, and LS). To check the model adequacy, the model's comparative fit, parsimony correction, and absolute fit were assessed using the goodness-of-fit indices commonly used in the SEM technique, including the comparative fit index (CFI) and Tucker-Lewis index (TLI), the root mean square error of approximation (RMSEA), and root mean square residual (MRSR). According to $\mathrm{Hu}$ and Bentler's principle [49], the model is considered as having an adequate fit with the data when the CFI and TLI are $>.90$, the RMSEA $<0.06$, and SRMR $<.08$.

For the SEM estimation, data merged across the two waves of the survey was used for model fitting. Sociodemographic characteristics of the sample and their scores of $S R H, S S$ and $L S$ across both waves are reported in Table 1. Samples' equivalised monthly household income was derived from dividing their monthly household income to the square root of the household size. Also, the second wave sample's monthly household income was deflated by the consumer price index for a valid reflection of the real changes of household income. Following convention, standardised coefficients of the cross-lagged model in SEM were reported.

Table 1 Sample's socioeconomic characteristics and descriptive statistics on the main variables of interest $(\mathrm{N}=1,109)$ was to estimate the WTPs of $S R H$ and $S S$ on $L S$. Specifically, the estimated causal effects $(\beta \mathrm{c})$ of $S R H_{1}$ and $S S_{1}$ on $L S_{2}$ as well as $H I_{1}$ on $L S_{2}$ were used for computing the marginal rate of substitutions of $S R H$ and $S S$ with respect to $H I$, respectively. Subsequently, it is substituted into the formulae of compensating surplus [21] expressed as, 


$$
\bar{w}-e^{\left[\ln (\bar{w})-\frac{\beta a}{\beta_{b}} \Delta x\right]},
$$

where $\bar{w}$ is sample's median equivalised monthly household income, $\beta_{a} / \beta_{b}$ was the marginal rate of substitution, and $\Delta x$ is the change in the non-market condition (i.e., $S R H$ or $S S$ ). Estimates yield from

222 the formulae reflects the WTP of the condition, which can be interpreted as (hypothetically) the amount of additional equivalised monthly household income required to equate the changes in life satisfaction in relation to the increase or decline either in $S R H$ or $S S$.

225

226

\section{Results}

\section{Cross-lagged analysis of the relationship between SRH, SS, HI, and LS}

228 Using the SEM technique, the hypothesised cross-lagged model denoting the complex relationships among $S R H, S S, L S, H I$, and the socioeconomic variables as covariates were examined. Model diagnostics suggested that there was an adequate fit between the specified model and the empirical data, as all the goodness-of-fit indices met the Hu and Bentler's threshold (CFI $=.93$, TFI $=.92$, RMSEA $=.05, \mathrm{SRMR}=.07)$.

Figure 1 summarises the major findings of the cross-lagged analysis on the relationships among $S R H, S S, H I$, and $L S$. There are a number of noteworthy observations. First, the model indicated that there were strong causal associations among the four variables across the timepoints (the curved arrows in Figure 1; e.g., $\mathrm{HI}_{1} \square \mathrm{HI}_{2}: \beta=0.458,95 \% \mathrm{CI}$ : $\left.0.296,0.619\right)$. The model also pointed to some significant cross-sectional correlations between the variables of SRH, SS and HI, and LS at each wave (i.e., $S R H_{1}$ $\leftrightarrow S S_{1}: \beta=0.107,95 \% \mathrm{CI}: 0.037,0.196 ; \mathrm{SRH}_{2} \leftrightarrow \mathrm{SS}_{2}: \beta=0.122,95 \% \mathrm{CI}: 0.049,0.199 ; H I_{2} \leftrightarrow L S_{2}: \beta$ $=0.101,95 \%$ CI: $0.042,0.160$, not shown in Figure 1 for the purpose of parsimony). Second, mutual causality was detected among the relationships between $S R H$ and $L S$ and between $H I$ and $L S$.

241 Specifically, the model indicated the casual effects of $S R H_{1}$ and $H I_{1}$ on $L S_{2}$ were significant; one 242 standard deviation increases in $S R H$ and $H I$ were casually associated with a standard deviation increase in $L S$ by the unit of 0.078 (95\%CI: $0.020,0.151)$ and 0.061 (95\%CI: $0.009,0.112)$, respectively. At the 
magnitude of the estimates, the reverse causal effects of $L S_{1}$ on $S R H_{2}$ and $H I_{2}$ were even greater than

246 their respective causal effects (the effect of $L S_{1}$ on $S R H_{2}, \beta=0.086,95 \%$ CI: 0.020, 0.151; the effect of $247 L S_{1}$ on $\left.H I_{2}, \beta=0.073,95 \% \mathrm{CI}: 0.020,0.151\right)$. For the relationship between $S S$ on $L S$, while the causal 248 influence of $S S$ on $L S$ was found to be significant $(\beta=0.083,95 \%$ CI: $0.014,0.152)$, its respective reverse causal effect was however not detected. Overall, $R^{2}$ suggested the model accounted for $45 \%$, of the variance of the variables of $L S_{2}$.

Fig. 1 The causal and reverse causal effects of household income, self-rated health, and social support on life satisfaction

254

255

\section{The implicit WTPs of SRH and SS on LS}

256

Based on the results of the cross-lagged analysis (Figure 1), the marginal rate of substitution of $S R H$ and $S S$ on $L S$ were estimated to be 1.28 (95\%CI: $0.43,2.15)$ and 1.36 (95\%CI: $0.23,2.49)$, respectively. This is interpreted as respondents' increase (decrease) in $L S$ owing to their improvement (deterioration) in $S R H$ and $S S$ is equivalent to the effect of increasing (reducing) their $H I$ by 1.28 and 1.36 unit of standard deviation respectively. Substituting it into the formulae of compensating surplus, the implicit WTPs of improving the sample's $S R H$ from "poor health" to "excellent health" and enhancing their $S S$ from "little support" to "a lot of support" are thus equivalent to an additional increase in their equivalised monthly household income by $\operatorname{HK} \$ 56,000(95 \% \mathrm{CI}$ : $\operatorname{HK} \$ 26,900$, $\operatorname{HK} \$ 68,600)$ and HK\$39,400 (95\%CI: HK\$10,800, HK\$48,300), respectively. ${ }^{1}$

\section{Discussion}

267 Drawing data from a representative panel survey in Hong Kong, this study examined the causal effects of change in self-rated health and social support on individuals' overall satisfaction with life. Adopting the wellbeing valuation method $[13,16,17]$, this study expressed these causal effects in an economic

\footnotetext{
${ }^{1}$ Estimation of the WTPs of SRH and SS were based on the formulae of compensating surplus. In this estimation, $\bar{w}$ is the sample's equivalised monthly $H I$ : $\mathrm{HK} \$ 14.2 \mathrm{k}$.
} 
term (i.e., WTPs). To the best of the author's knowledge, this is the first to derive the shadow price of

271 self-rated health status and social support in a predominantly Chinese society. It extends the wellbeing

272 valuation literature on health and social outcomes as it is one of the few conducted in an Asian context.

273 This research has implications for the practice of social impact evaluation. Specifically, the

274 derived shadow price of self-rated health and social support provide ways to tie social impact evaluation

275

276

277 with some forms of cost-benefit analysis (e.g., social return on investment), which is a tool where policymakers commonly relied on for decision making [5]. Currently, in the absence of culturallyattuned economic proxies, practice among social impact evaluators sometimes apply economic proxies derived from other socio-cultural context (e.g., Western settings) to monetise social non-market outcomes, despite the notable cultural difference on individuals' evaluation on domains of life [50,51]. This study offers a way to make socio-cultural embedded cost-impact assessment plausible in the Chinese context.

Findings of the cross-lagged analysis illustrated that the relationships among the examined variables are complex. First, the model reflected the existence of significant autoregressive correlations within each main variable of interest (i.e., SRH, SS, HI, and LS). This is fairly intuitive given the timeframe between two data collection timepoints was not long (i.e., approximately twelve months). In addition, cross-sectional correlations among the variables of interest were also detected (e.g., between SRH and SS). The finding is in line with the domains-of-life literature highlighting the intertwining nature among individuals' various aspects of life [31]. Furthermore, the results not only showed the existence of the causal influences of self-rated health, social support, and equivalised monthly household income on samples' satisfaction with life, it also suggested that the reverse causal mechanisms among these variables were significant. Particularly, the sample's baseline life satisfaction was positively and casually associated with their equivalised monthly household income and self-rated health, respectively. Previous research has illustrated that individuals with greater life satisfaction are more productive and are more likely to be promoted and hence may earn a higher income $[20,52,53]$. Positive affect has also been shown to improve the immune system and reduce a person's susceptibility to illness $[54,55]$. Taken together, results of this study, on the one hand, add to a large pool of literature illustrating individual's wellbeing as constitutes of multiple areas of life, it nevertheless reflects the 
plausibility of the situational mechanism in which one's overall wellbeing affects their different spheres 299 of life [30].

The existence of the reverse causal influences existed in the relationship between sample's HI and

301 LS and between SRH and LS further affirms the need to attend the endogeneity problem in the regression analysis, in order to avoid an overestimation of the implicit monetary value of health and social outcomes $[27,28]$. In addition, this study also shows collinearity likely exists between exogenous variables (i.e., social and health conditions), it serves as a reminder for researchers to attend to this problem in future wellbeing valuation studies. The cross-lagged approach simultaneously addresses the cross-sectional associations between the exogenous variables and the reverse-causal influences could be a plausible way to minimise the endogeneity issue in this line of valuation work. support on individual's life satisfaction in predominantly Chinese society. Also, unlike some prior work, this study employed statistical procedure in order to minimise potential bias in the estimation process arising from the endogeneity issue. However, it is not without limitations. First, this study was secondary data analysis, and hence the selection of variables for model estimation was restricted by the original survey design. Second, although it is aware that the causal relationship between $S R H, S S$ and

$314 L S$ may vary across age and sex [56], this study, however, did not perform sub-demographic analysis owing to a limited power due to the restricted sample size. While the WTPs estimated in this study may reflect the implicit monetary value of self-rated health and social support among the general population, caution should be exercised when generalising it to particular sub-demographic groups. Further research

318 is warranted to estimate the variation. Third, as in almost all wellbeing valuation analysis, it cannot rule 319 out the possibility that endogeneity still existed in the estimates, as the problem can arise from other sources (e.g., measurement error) that may not be able to be controlled from a statistical standpoint. 


\section{Conclusions}

323 This study offers a way to monetise the impact of increasing individuals' self-rated health status and

324 social support on their overall satisfaction with life. It has practical implications for the use of social

325 cost-benefit analysis in assessing wellbeing policy initiatives for the Chinese population.

326

327 Supplementary Information

328 Additional file 1: Implicit WTPs of self-rated health status and social support from wellbeing valuation 329 studies in an Asian context.

330

331 Abbreviations

332 SRH: Self-rated health; SS: Social Support; CI: Confidence Interval; WTP: willingness-to-pay; LS:

333 Life Satisfaction; HI: Household Income; SEM: Structural Equation Modelling; CFI: Comparative Fit

334 Index; TLI: Tucker-Lewis Index; RMSEA: Root Mean Square Error of Approximation; MRSR: Root

335 Mean Square Residual

336

\section{Declarations}

338 Ethics approval and consent to participate

339 The ethical considerations were approved by the Human Research Ethics Committee, the University of

340 Hong Kong (HREC Reference Number: EA1506006). The author declares that all methods were

341 performed in accordance with the relevant guidelines and regulations. Informed consent was obtained

342 from all individual participants included in the study.

344 Consent for publication

345 Not applicable. 
348 The datasets used and/or analysed during the current study available from the corresponding author on

349 reasonable request.

350

351 Competing interests

352 The author declares that he/she has no competing interests.

353

$354 \quad$ Funding

355 No funding was received.

356

357 Author's contributions

358 CHC conceived of the study, designed, executed the statistical analyses, and drafted the manuscript.

359

360 Acknowledgements

361 Not applicable.

362

363 Author's information

364 Not applicable.

365

366 References

367 1. Adler A, Seligman ME: Using wellbeing for public policy: Theory, measurement, and

368 recommendations. International journal of wellbeing 2016, 6(1).

369 2. Diener E, Lucas R, Helliwell JF, Helliwell J, Schimmack U: Well-being for public policy:

$370 \quad$ Series in Positive Psychology; 2009.

371 3. Kittiprapas S: Subjective-well-being: New paradigm for measuring progress and public policies. In: 3rd OECD World Forum, Korea: 2009; 2009.

373 4. O’Donnell G, Deaton A, Durand M, Halpern D, Layard R: Wellbeing and policy. 2014. 
374 5. Fuguitt D, Wilcox SJ: Cost-benefit analysis for public sector decision makers: Greenwood $375 \quad$ Publishing Group; 1999.

376 6. Johannesson M, Jönsson B: Economic evaluation in health care: is there a role for cost377 benefit analysis? Health policy 1991, 17(1):1-23.

378 7. Brouwer R, Job FC, van der Kroon B, Johnston R: Comparing willingness to pay for improved drinking-water quality using stated preference methods in rural and urban Kenya. Applied health economics and health policy 2015, 13(1):81-94.

8. Gries KS, Regier DA, Ramsey SD, Patrick DL: Utility Estimates of Disease-Specific Health States in Prostate Cancer from Three Different Perspectives. Applied Health Economics and Health Policy 2017, 15(3):375-384.

9. Klose T: The contingent valuation method in health care. Health policy 1999, 47(2):97123.

10. Settumba SN, Shanahan M, Botha W, Ramli MZ, Chambers GM: Reliability and validity of the contingent valuation method for estimating willingness to pay: A case of in vitro fertilisation. Applied health economics and health policy 2019, 17(1):103-110.

11. Dolan P, Kahneman D: Interpretations Of Utility And Their Implications For The Valuation Of Health. The Economic Journal 2008, 118(525):215-234.

12. Freeman III AM, Herriges JA, Kling CL: The measurement of environmental and resource values: theory and methods: Routledge; 2014.

13. Frey BS, Luechinger S, Stutzer A: Valuing public goods: The life satisfaction approach. 2004.

14. Mühlbacher A, Johnson FR: Choice experiments to quantify preferences for health and healthcare: state of the practice. Applied health economics and health policy 2016, 14(3):253-266.

15. Walasek L, Brown GDA, Ovens GD: Subjective well-being and valuation of future health states: Discrepancies between anticipated and experienced life satisfaction. Journal of Applied Social Psychology 2019, 49(12):746-754. 
401 16. Frey BS, Stutzer A: The use of happiness research for public policy. Social choice and $402 \quad$ welfare 2012, 38(4):659-674.

403 17. Welsch H: Environment and happiness: Valuation of air pollution using life satisfaction 404 data. Ecological economics 2006, 58(4):801-813.

405 18. Pavot W, Diener E: The satisfaction with life scale and the emerging construct of life 406 satisfaction. The journal of positive psychology 2008, 3(2):137-152.

19. Kahneman D, Sugden R: Experienced utility as a standard of policy evaluation. Environmental and resource economics 2005, 32(1):161-181.

20. Brown TT: The Subjective Well-Being Method of Valuation: An Application to General Health Status. Health services research 2015, 50(6):1996-2018.

21. Ferreira S, Moro M: On the use of subjective well-being data for environmental valuation. Environmental and Resource Economics 2010, 46(3):249-273.

22. Powdthavee N: Putting a price tag on friends, relatives, and neighbours: Using surveys of life satisfaction to value social relationships. The Journal of Socio-Economics 2008, 37(4):1459-1480.

23. Powdthavee N, Van Den Berg B: Putting different price tags on the same health condition: Re-evaluating the well-being valuation approach. Journal of health economics 2011, 30(5):1032-1043.

24. Clark AE, Oswald AJ: A simple statistical method for measuring how life events affect happiness. international Journal of Epidemiology 2002, 31(6):1139-1144.

25. Chandoevwit W, Thampanishvong K: Valuing social relationships and improved health condition among the Thai population. Journal of happiness studies 2016, 17(5):2167-2189. The life satisfaction approach. Journal of Happiness Studies 2017, 18(5):1275-1303. 
428 28. Ambrey CL, Fleming CM: The causal effect of income on life satisfaction and the

429 implications for valuing non-market goods. Economics Letters 2014, 123(2):131-134.

430 29. Van Praag BM, Van Praag B, Ferrer-i-Carbonell A: Happiness quantified: A satisfaction

$431 \quad$ calculus approach: Oxford University Press; 2004.

432 30. Rojas M: Life satisfaction and satisfaction in domains of life: Is it a simple relationship? $433 \quad$ Journal of happiness studies 2006, 7(4):467-497.

434 31. Rojas M: The complexity of well-being: A life-satisfaction conception and a domains-oflife approach. Researching well-being in developing countries: From theory to research 2007:259-280.

32. Diener E, Suh EM, Lucas RE, Smith HL: Subjective well-being: Three decades of progress. Psychological bulletin 1999, 125(2):276.

33. Cummins RA: The domains of life satisfaction: An attempt to order chaos. In: Citation classics from social indicators research. edn.: Springer; 2005: 559-584.

34. Argyle M: The psychology of happiness: Routledge; 2013.

35. Gleicher Y, Croxford R, Hochman J, Hawker G: A prospective study of mental health care for comorbid depressed mood in older adults with painful osteoarthritis. BMC Psychiatry

36. Ekström M, Johansson B: Talk scandals. 2008, 30(1):61-79.

37. Williamson GM, Shaffer DR, Schulz RJHP: Activity restriction and prior relationship history as contributors to mental health outcomes among middle-aged and older spousal caregivers. 1998, 17(2):152.

38. Bai X, Yang S, Knapp M: Sources and directions of social support and life satisfaction 7(9):e013228. 
40. Peng C, Fang L, Wang JS-H, Law YW, Zhang Y, Yip PS: Determinants of Poverty and Their Variation Across the Poverty Spectrum: Evidence from Hong Kong, a HighIncome Society with a High Poverty Level. Social Indicators Research 2019, 144(1):219250.

41. DeSalvo KB, Fisher WP, Tran K, Bloser N, Merrill W, Peabody J: Assessing measurement properties of two single-item general health measures. Quality of Life Research 2006, 15(2):191-201.

42. Nakata A, Takahashi M, Swanson N, Ikeda T, Hojou M: Active cigarette smoking, secondhand smoke exposure at work and home, and self-rated health. Public Health 2009, 123(10):650-656.

43. Heaney CA, Israel BA: Social networks and social support. Health behavior and health education: Theory, research, and practice 2008, 4:189-210.

44. Sachs J: Validation of the satisfaction with life scale in a sample of Hong Kong university students. Psychologia 2003, 46(4):225-234.

45. Martens EP, Pestman WR, de Boer A, Belitser SV, Klungel OH: Instrumental variables: application and limitations. Epidemiology 2006:260-267.

46. Giovanis E, Ozdamar O: STRUCTURAL EQUATION MODELLING AND THE CAUSAL EFFECT OF PERMANENT INCOME ON LIFE SATISFACTION: THE CASE OF AIR POLLUTION VALUATION IN SWITZERLAND. 2016, 30(3):430-459.

47. Hamaker EL, Kuiper RM, Grasman RP: A critique of the cross-lagged panel model. Psychological methods 2015, 20(1):102.

48. Powdthavee N, Wooden M: Life satisfaction and sexual minorities: Evidence from Australia and the United Kingdom. Journal of Economic Behavior \& Organization 2015, 116:107-126.

49. Hu L-t, Bentler PM: Fit indices in covariance structure modeling: Sensitivity to underparameterized model misspecification. Psychological methods 1998, 3(4):424.

50. Diener E, Diener M: Cross-Cultural Correlates of Life Satisfaction and Self-Esteem. Journal of Personality and Social Psychology 1995, 68(4):653-663. 
483 51. Mikucka M: Does Individualistic Culture Lower the Well-Being of the Unemployed?

$484 \quad$ Evidence from Europe. J Happiness Stud 2014, 15(3):673-691.

485 52. Achor S: The happiness advantage : the seven principles of positive psychology that fuel 486 success and performance at work, 1st ed. edn. New York: Crown Business; 2010.

487 53. O'Donnell G, Oswald AJ: National well-being policy and a weighted approach to human 488 feelings. Ecological Economics 2015, 120:59-70.

489 54. Burger J, Caldwell D: Personality, Social Activities, Job-Search Behavior and Interview 490 Success: Distinguishing Between PANAS Trait Positive Affect and NEO Extraversion. $491 \quad$ Motivation and Emotion 2000, 24(1):51-62.

492 55. Diener E, Seligman MEP: Very Happy People. Psychological Science 2002, 13(1):81-84.

493 56. Cheung F, Lucas RE: When Does Money Matter Most? Examining the Association

494 Between Income and Life Satisfaction Over the Life Course. Psychology and Aging 2015, $495 \quad 30(1): 120-135$.

496 


\section{Figures}

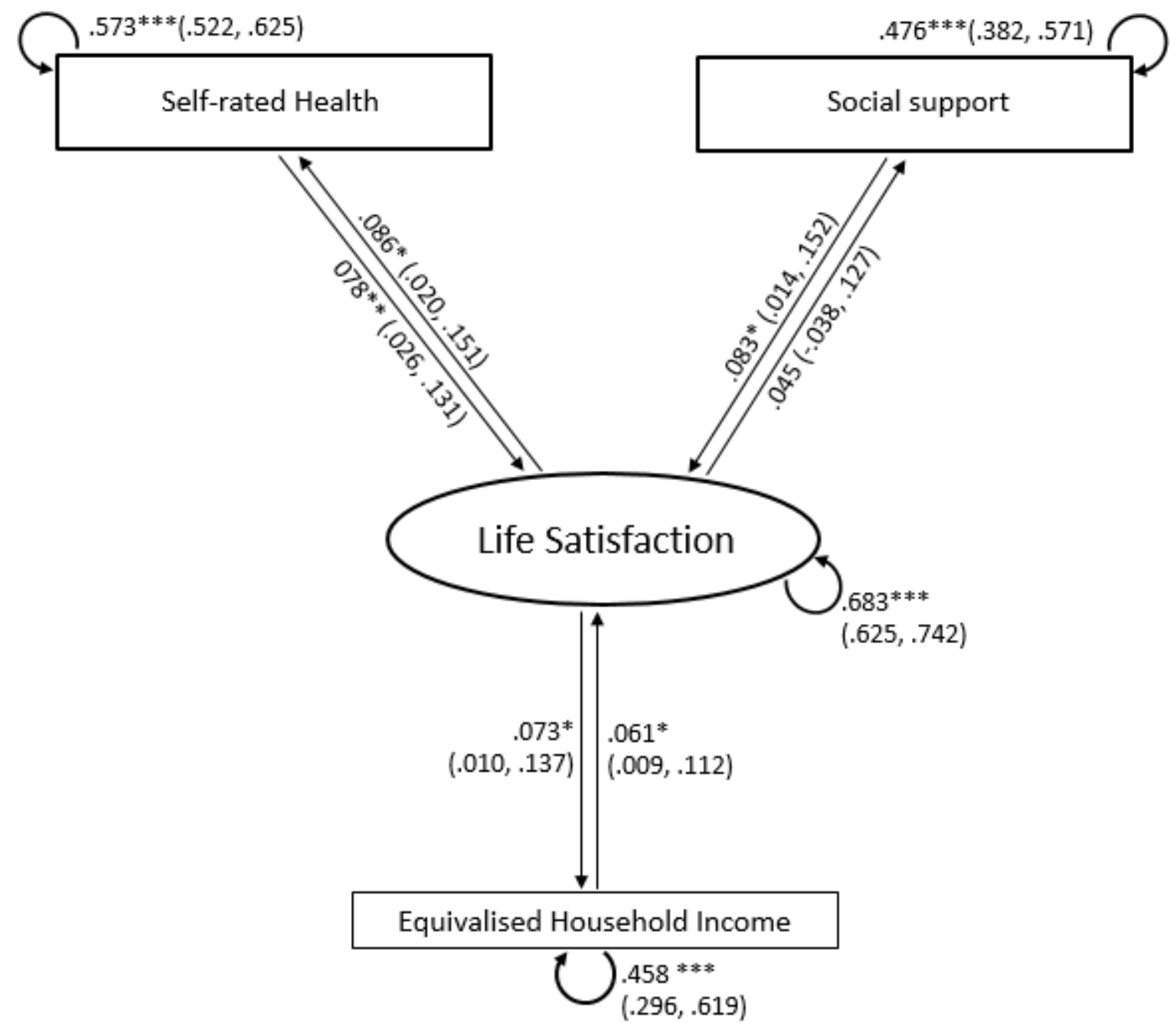

\section{Figure 1}

The causal and reverse causal effects of household income, self-rated health, and social support on life satisfaction

\section{Supplementary Files}

This is a list of supplementary files associated with this preprint. Click to download.

- Additionalfile1.docx 